\title{
Protée
}

\section{Une rencontre singulière entre structuralisme et bouddhisme : Saussure, Bouddha, Lévi-Strauss}

\section{Sungdo Kim}

Volume 39, numéro 2, automne 2011

URI : https://id.erudit.org/iderudit/1007165ar

DOI : https://doi.org/10.7202/1007165ar

Aller au sommaire du numéro

Éditeur(s)

Département des arts et lettres - Université du Québec à Chicoutimi

\section{ISSN}

0300-3523 (imprimé)

1708-2307 (numérique)

Découvrir la revue

Citer cet article

Kim, S. (2011). Une rencontre singulière entre structuralisme et bouddhisme : Saussure, Bouddha, Lévi-Strauss. Protée, 39(2), 19-29.

https://doi.org/10.7202/1007165ar
Résumé de l'article

Dans cette étude, en choisissant Saussure et Lévi-Strauss comme les figures exemplaires du mouvement structuraliste, j'ai tenté de faire un rapprochement philosophique entre le bouddhisme et le structuralisme et d'expliciter les raisons de ce rapprochement en me concentrant sur certains thèmes majeurs. Cette analyse comporte deux parties. En premier lieu, sur le plan historique, j'ai fourni des éléments méthodologiques de la comparaison des pensées lointaines et rappelé les indices historiques de la réception européenne du bouddhisme, de l'époque de Saussure à celle de Lévi-Strauss, pour saisir la profondeur et la nature du bouddhisme dans les sciences humaines européennes. Dans un deuxième temps, sur le plan ontologique, j'ai essayé d'analyser en parallèle le rejet des ontologies substantielles et la déconstruction du moi et du sujet de trois figures, Bouddha, Saussure, Lévi-Strauss, en mettant en relief trois éléments : vacuité, critique du moi, décentrement de l'anthropocentrisme. 


\section{UNE RENCONTRE SINGULIÈRE ENTRE STRUCTURALISME ET BOUDDHISME: SAussure, BOUdDHA, LÉVI-STRAuss}

SUNGDO KIM

\section{INTRODUCTION}

Il me semble encore prématuré de tenter de concevoir une étude complète et solide sur les affinités ou les similarités conceptuelles entre le structuralisme et le bouddhisme. Ce jugement peut se justifier par plusieurs raisons circonstancielles. Tout d'abord, on est confronté à une pénurie de recherches de base pouvant servir de repérage dans de telles investigations. Il faut que nous possédions déjà quelques références fondamentales permettant d'évaluer l'envergure de l'impact que le bouddhisme a eu sur les sciences humaines françaises pendant le XXe siècle. Mais, à ma connaissance, une telle étude de synthèse n'existe pas. Bien sûr, je ne compte pas dans cette catégorie tous les essais qui ont traité les divers aspects culturels et sociologiques de l'introduction du bouddhisme en Europe, en France en particulier, depuis quelques décennies (Obadia, 1999) ou qui ont décrit, dans une perspective critique, une histoire de la réception du bouddhisme en Occident depuis trois siècles en en retraçant les diverses phases (Lenoir, 1999). Par exemple, dans ses travaux très accessibles, RogerPol Droit a bien montré que, pour les grands penseurs européens comme Hegel, Nietzsche ou Renan, l'essentiel de l'enseignement bouddhique a été interprété comme synonyme de négation de la vie, d'anéantissement de l'individu et de "culte du néant", en déconstruisant l'imaginaire de l'Occident qui a mis en scène son propre nihilisme (Droit, 1997). Il avait déjà révélé la paradoxale amnésie qui caractérisait la réception occidentale à l'égard des pensées indiennes, et du bouddhisme en particulier (Droit, 1989). Cette amnésie est multiple et complexe sur les plans historique, épistémologique et culturel. L'auteur signale au premier chef l'absence d'intérêt de la philosophie française du XXe siècle pour l'Inde, se demandant quelle en est la cause, sans pouvoir y apporter de réponse. Selon lui, «[à] propos de l'Inde, les philosophes d'aujourd'hui sont entrés dans le monde du silence» (ibid.: 24).

Tout en appréciant la valeur scientifique de ces travaux qui ont bien réussi à résoudre des problèmes déterminants dans l'histoire de cette découverte et de cet oubli de l'Inde par la philosophie européenne, je voudrais simplement attirer l'attention sur un fait (non négligeable). En effet, parmi les grands noms de la pensée française moderne - Merleau-Ponty, Deleuze, Lacan, Foucault - qui se sont intéressés à la civilisation et à la pensée de l'Extrême-Orient, notamment de l'Inde, on constate une omission grave concernant notre sujet: pas une seule mention du nom de Lévi-Strauss dans la totalité de l'ouvrage. 
Dans cette histoire culturelle passionnante de la découverte du bouddhisme par l'Occident, il est important de noter la coupure épistémologique qui a fait naître le bouddhisme comme objet scientifique, laquelle remonte aux alentours de 1830 selon Lubac (1952: 132-148). Je me permets ici de présenter une description révélatrice d'un grand bouddhologue à propos de ce processus de démythologisation et de ses effets embarrassants:

Le bouddhisme "oriental» (indien) allait de la sorte être revu et corrigé par la «bouddhologie» occidentalisée de façon à présenter les diverses écoles bouddhiques comme autant de "philosophies» parfaitement démythologisées. En ce sens, le bouddhisme "philosophique» est lui-même un mythe. Mais le bouddhisme "mythologique», lui, est à bien des égards une philosophie de la vie. Cette dimension disparaît lorsqu'on réduit trop vite la doctrine bouddhique à son contenu philosophique, au détriment de ses aspects littéraires et symboliques. Ce type de rationalisme occulte l'entrelacement des systèmes textuels, méditatifs et rituels au sein de la tradition. (Faure, 1998: 19)

Au fond, malgré une longue histoire de la réception du bouddhisme en Occident, une déclaration faite par un excellent historien du bouddhisme au milieu du XXe siècle est encore valide de nos jours: «le bouddhisme demeure une terra incognita" (Lubac, 1952: 261).

S'agissant des travaux qui traitent des liens épistémologiques ou historiques entre Saussure et le bouddhisme, d'une part, et Lévi-Strauss et le bouddhisme, d'autre part, on constate à la fois une insuffisance et une asymétrie à bien des égards. Un certain nombre d'études ont fait une tentative de rapprochement entre la pensée hindoue et le linguiste genevois dans une perspective de sémiolinguistique, que je présenterai plus en détail dans la section suivante. En revanche, concernant le lien entre le grand anthropologue et la pensée bouddhique, mis à part quelques travaux en langue anglaise, on constate une quasi-absence du côté des spécialistes français de Lévi-Strauss. Ce silence contraste avec des études menées dans différentes disciplines, telles que les sciences cognitives (Varela, 1991, 1992), la philosophie (Bonardel, 2008), la psychanalyse (Suzuki, Fromm et
Martino, 1971), la déconstruction (Wang, 2001) ou la critique littéraire, au sujet du rapprochement de leur domaine et du bouddhisme.

Enfin, je voudrais faire quelques remarques sur la nature et la limite de ce travail. Avant tout, il ne relève pas du genre de la philosophie comparée si, par cette discipline,

[...] on entend [...] ici un travail sérieux reposant sur une connaissance approfondie des langues philosophiques concernées [...] ainsi que des contextes culturels et religieux respectifs, et non des rapprochements syncrétiques et superficiels inspirés par l'impatience de retrouver partout et toujours une même et unique philosophia perennis.

(Lacrosse et alii, 2005: 7-8)

En vérité, l'auteur de ces lignes n'a ni les compétences pour lire les textes bouddhiques originaux, ni les connaissances approfondies de l'histoire du bouddhisme indien (Lamotte, 1976). Cela dit, mon savoir du bouddhisme d'ici s'appuie sur quelques traités d'introduction au bouddhisme pour les novices (Conze, 1951; Borges, 1979; Berval, 1987; Faure, 1998). Il ne s'agit pas non plus de déterminer, d'une façon précise, une dialectique des différences et des similitudes entre les deux chemins de pensée appartenant à des horizons linguistiques, chronologiques et géographiques distincts. Notre analyse pratique toutefois un certain comparatisme, mais ni purement d'étrangeté, ni simplement de ramification au sens de la distinction de Jullien (2005: 65-78). Je dirais que ce comparatisme est plutôt panchronique au sens saussurien. Pourtant, il faut reconnaître pleinement la possibilité d'appliquer un comparatisme de ramification si l'on se rappelle ces paroles de Nietzsche soulignant la généalogie systématique de tous les concepts philosophiques:

Les divers concepts philosophiques ne présentent rien

d'arbitraire, ils n'ont pas une croissance autonome, mais se développent apparentés les uns aux autres et en relations réciproques. [...] Ils n'en font pas moins partie d'un système, au même titre que toutes les espèces de la faune d'un continent. (1993: 575-576)

Il est ici nécessaire de préciser les caractéristiques et la pertinence de la comparaison en général. Les 
conditions de possibilité de la comparaison ne sont pas données naturellement, mais sont à élaborer et à construire à l'aide de certains critères. Il faut admettre qu'il y a toujours quelque chose d'arbitraire dans le montage comparatiste, quelque chose qui est imposé de l'extérieur. Mais l'important n'est pas tant l'arbitraire initial que la fécondité qui en ressort et le mode de pertinence qui en découle: pertinence historique, pertinence heuristique.

Une première approche consiste à postuler que le bouddhisme et le structuralisme, nonobstant des différences ou des variations de surface, se laissent identifier dans la mesure où ils semblent dire la même chose ou, du moins, partager les mêmes thématiques. Cependant, il faut se méfier d'une universalisation forcée et éclectique en se demandant :

[...] si l'idéal d'une synthèse unitaire fondée sur quelque nécessité universelle de l'esprit humain ou bien sur l'existence d'invariants transculturels ne repose pas sur des exigences sentimentales qui convergent vers un vague éclectisme spiritualiste. (Chenet, 2005: 82)

Or, il est une autre approche, plus modeste en apparence, qui tente de mettre en évidence l'existence de simples parallèles entre des philosophes relevant de traditions différentes. La mise en scène qui choisit le fondateur même du bouddhisme comme interlocuteur de Saussure et Lévi-Strauss rend l'entreprise comparatiste plus complexe en même temps qu'elle en rend plus perceptibles les enjeux. Par exemple, est-il permis ou sensé de comparer un fondateur de la linguistique moderne et un grand Sage de l'antiquité orientale? Existe-t-il pour cela un socle épistémologique commun, ou du moins un point d'intersection, réel ou apparent, entre les trois figures ici confrontées?

Dans les pages qui suivent, j'aimerais examiner les rapports possibles entre la généalogie structuraliste et le bouddhisme sous son aspect philosophique. Je n'entends nullement prôner une sorte de concordisme entre ces deux pensées si lointaines. Je cherche simplement à repérer dans la pensée structurale certains éléments plus ou moins dissimulés, qui nous rendraient peut-être plus accessible tel ou tel aspect du bouddhisme. Il s'agit de présenter des analogies inattendues ou autorisant des rapprochements de signification féconds. À travers ce type de comparatisme constructif, on peut espérer trouver un moyen de reformuler quelques problèmes classiques de l'épistémè structuraliste.

\section{SAUSSURE ET LE BOUDDHISME:} QUELQUES TRACES D'INFLUENCE ET D'AFFINITÉ

Les preuves biographiques qui témoignent de l'attention de Saussure pour le monde indien ont déjà été signalées par de Mauro (1995: 380). Mais, pour connaître la substance matérielle de cet intérêt saussurien pour le bouddhisme, il a fallu attendre la publication - bien qu'elle soit très partielle - des manuscrits de Harvard, tentée par H. Parret (1993) qui a réuni une vingtaine de pages concernant la théosophie et la mythographie indiennes. Je signale que la totalité de ces manuscrits restés inédits en majorité atteint presque 1000 pages, qui traitent des différentes thématiques de la phonétique à la métaphysique indiennes. En se basant sur ces matériaux, Rastier a identifié quelques points communs entre la sémiotique saussurienne et la pensée bouddhique, qui pourraient converger en deux ruptures, déontologie et négativité, et a noté que Saussure a rompu «le substantialisme ontologique de tradition aristotélicienne» (Rastier, 2003: 26). Ensuite, une indologiste, F. Atlali-Voisin, a procédé à l'examen des relations possibles entre Saussure et les divers domaines de l'indologie et suggéré une influence de la pensée grammairienne de l'Inde sur le doute ou les interrogations manifestées dans les Écrits de linguistique générale et les Manuscrits de Harvard:

Si notre thèse s'avère pertinente, l'érudition de Ferdinand de Saussure concernant l'Inde et la réflexion souterraine qu'il semble avoir eue à son propos révéleront une pensée linguistique nourrie pour une part, de la philosophie de la parole de l'époque védique et brahmanique.

(Atlani-Voisin, 2003: 80)

Enfin, je tiens à signaler l'existence de deux études plus techniques: l'une tente d'analyser en parallèle la théorie de l'apoha et la théorie saussurienne et de statuer sur l'hypothèse d'éventuelles interférences indologiques dans la genèse et dans le développement 
de la pensée saussurienne (D'Ottavi 2010: 169); l'autre traite une comparaison sémiotique entre le signe saussurien et le nama-rupa du sanscrit (Small, 1987).

Notre lecture des manuscrits confirme quelques preuves bibliographiques. En premier lieu, Saussure a lu les ouvrages magistraux et des articles techniques de l'indologie de son temps. Il suffirait d'en mentionner quelques-uns parmi d'autres: Les Religions de l'Inde par Auguste Barth en 1879, Introduction à l'histoire $d u$ bouddhisme indien d'Eugène Burnouf en 1844 (1876), un ouvrage pionnier de l'histoire des études bouddhiques en Occident, La Religion védique d'après les hymnes du Rig-Véda d'Abel Bergaigne (voir Amacker, 1988) et d'autres grands noms comme Klaproth et Rémusat. En effet, le maître genevois a manifesté un intérêt tout particulier pour les divers thèmes de l'indologie, de la théosophie aux légendes et aux mythes indiens incluant le Véda, qui dépassent largement les domaines locaux de la grammaire sanscrite, dévoilant ainsi ses connaissances profondes et érudites sur la matière, contrairement à sa déclaration de modestie: "Je suis très peu versé dans la littérature sanscrite. Simplement occupé d'études linguistiques, je n'ai effleuré l'Inde qu'au point de vue de sa langue" (dans Parret, 1993: 223). Plus concrètement, Saussure a essayé de rédiger la préface du premier volume du livre de Paul Oltremare, son collègue universitaire, Histoire des idées théosophiques dans l'Inde, bien qu'il n'ait pu la terminer.

Les thématiques philosophiques développées dans ces manuscrits sont les suivantes: la découverte de l'Inde en Occident, l'absence du genre historique chez les Hindous, la théosophie, l'essence de la pensée hindoue, la substance et les formes, le karma, le Moi.

Ce qui est ici remarquable, c'est que Saussure a bien saisi le caractère hybride de la pensée hindoue, y compris le bouddhisme, en désignant les peuples de l'Inde comme «les premiers rêveurs du monde sur la condition des êtres":

Mais de quoi s'agit-il? De solutions religieuses? Non [...] Philosophiques? Pas absolument non plus... (Quelque chose de mixte entre la croyance et le produit de la pensée réfléchie, sans que ni la croyance implique adhésion à un credo religieux ni la pensée indépendante n'arrive à être une philosophie parfaitement dégagée). (Cité dans Parret, 1993: 218)1 ${ }^{1}$
Ensuite, Saussure indique l'aspect non axiomatique de la théosophie hindoue comme suit: On ne peut pas parler de valeur axiomatique de ces choses pour le théosophe hindou parce qu'un axiome [...] ne peut avoir ce caractère que lorsqu'on fait 3 ou 4 efforts dont le premier consiste à l'énoncer, les autres à fixer sa place dans le système de la certitude. Mais jamais ces choses ne furent axiomatisées, et c'est par quoi que l'on voit qu'une obscure mentalité de race, qui n'est jamais parvenue à se reconnaître elle-même, est au fond de cette orgueilleuse philosophie fondée sur le moi. (Ibid.)

À ce propos, il est hautement intéressant de comparer ce passage avec la Note Item suivante déclarant la non-axiomaticité du langage:

Ne parlons ni d'axiomes, ni de principes, ni de thèses. Ce sont simplement et au pur sens étymologique des aphorismes, des délimitations - des limites entre lesquelles se retrouve constamment la vérité. (Engler, 1990: 42)

Au sujet de cette note, J. Fehr propose une interprétation très juste selon laquelle il faut remarquer une retenue ou une prudence, voire un doute face à ses propres énoncés que Saussure vient d'émettre. Le linguiste se refuse à les entendre aux sens d'axiomes, de principes, de thèses;

[...] il s'agit bien plutôt pour lui de délimiter ce domaine

à l'intérieur duquel il y a dans toute sa généralité sens et pertinence à se mettre en quête d'une vérité. (1997: 210)

Quant à Parret qui a suggéré de saisir la nature de l'instinct de Saussure en soulignant le caractère non domestiqué, subversif et liminaire du terrain saussurien, il énonce le commentaire suivant: «Saussure creuse son "lieu de vérité" dans un territoire immense, opaque, chaotique, et démarque, délimite ainsi un domaine» (2003: 64). Pour ma part, j'aimerais dire qu'il est possible de trouver dans ces deux passages une affinité entre le geste aphoristique de Saussure et le système la pensée théosophique indienne.

Mis à part cette affinité métaphysique, je voudrais évoquer une autre affinité entre la pensée saussurienne et le bouddhisme qui ont tous deux manifesté une rupture avec le substantialisme ontologique de la 
métaphysique occidentale. Dans la terminologie bouddhique, il s'agit du grand principe de la vacuité (śúnyatā en sanscrit). Selon Conze, elle exprime «la négation complète de ce monde par l'exercice de la sagesse" (1975: 132). Autrement dit, il s'agit du retrait total et de la libération absolue du monde qui nous entoure dans tous ses aspects. Le bouddhisme nous a présenté cette vérité de l'insubstantiel et du vide de toutes les formes de l'existence. Selon la Doctrine du Bouddha, ce que nous appelons notre existence n'est en réalité rien d'autre qu'un simple processus de phénomènes physiques et mentaux, processus qui se déroule depuis des temps immémoriaux, est antérieur à notre naissance apparente et se poursuivra après notre mort, à l'infini. «C'est l'ontologie tout entière, la croyance en l'être et en la substance, qui défaille» (Berval, 1987: 171).

En d'autres termes, derrière les apparences des formes, des couleurs, des sons, des sensations de la température et de l'espace, il n'y a rien. Il s'agit d'un «idéalisme absolu» où se montre «l'irréalité totale de l'univers» (Borges, 1979: 79, 91).

Saussure se rapproche de cette pensée bouddhique lorsqu'il se préoccupe de la question majeure de l'identité sémiotique et qu'il parvient à renier l'existence de l'identité linguistique et légendaire.

Il est vrai qu'en allant au fond des choses, on s'aperçoit dans ce domaine, comme dans le domaine parent de la linguistique, que toutes les incongruités de la pensée proviennent d'une insuffisante réflexion sur ce qu'est l'identité, [...] lorsqu'il s'agit d'un être inexistant comme le mot ou la personne mythique ou une lettre de l'alphabet, qui ne sont que différentes formes $d u$ SIGNE, au sens philosophique.

(Cité dans Godel, 1957b: 136)²

Dans ses recherches sur la légende germanique et les paradoxes relatifs au signe de la légende, ce doute se radicalise encore pour conclure à la non-existence du signe et constater simplement ses éléments constitutifs:

Le nom à lui seul ne signifie rien, c'est certain. Sont-ce donc les actes $d u$ personnage, ou son caractère, ou son entourage, ou quoi encore qui constituent le critère de l'identité? C'est un peu tout cela et rien de tout cela parce que tout peut avoir été à la fois transformé et transporté de A à B. Plus on étudiera la chose, plus on verra que la question n'est même pas de savoir où réside plutôt qu'ailleurs l'identité, mais s'il y a un sens quelconque à en parler.

(Cité dans Marinetti et Meli, 1986: 312)

Saussure avoue que les signes que sont les personnages de la légende et les lettres de l'alphabet n'ont jamais de consistance matérielle, c'est-à-dire que leur être est par essence fugitif et instable. Selon l'expression de Saussure lui-même, l'«association - que nous chérissons parfois - n'est qu'une bulle de savon" (ibid.: 192). Ni fantôme, ni bulles de savon, peut-être, dans la mesure où la bulle de savon "possède du moins son unité physique et mathématique" (ibid.), tandis que le signe ne consiste en rien. Je suis entièrement d'accord avec Arrivé, lorsqu'il affirme: «Il ne tient qu'à la rencontre provisoire et accidentelle de quelques traits voués à tout instant à se désunir» (2007: 96).

On pourrait multiplier les passages tirés des écrits saussuriens qui font penser au rapprochement avec la philosophie bouddhique de la vacuité. Par exemple, étant donné que les modifications des signes linguistiques et des signes légendaires sont données comme «incalculables», et bien que ceux-ci soient les uns et les autres de la même étoffe, les signes de la langue sont qualifiés non pas d'êtres inexistants, mais de termes en soi nuls:

Item. Il y a défaut d'analogie entre la langue et toute autre chose humaine pour deux raisons. 1/La nullité interne des signes. 2/La faculté de notre esprit de s'attacher à un terme en soi nul. (Engler, 1974-75: 29)

De la même manière, selon le bouddhisme,

[...] ce que nous désignons par les vocables "être», "individu», ou "personne», "moi» ou «lui», n'est rien d'autre qu'une combinaison changeante de phénomènes physiques et psychiques, et n'a pas d'existence en soi.

(Berval, 1987: 111-112)

On retrouve chez Saussure le principe similaire selon lequel la prétendue substance n'est qu'une collection de mutations et d'accidents. À ce propos, la discussion de Saussure sur l'identité personnelle et sur le non-moi est hautement intéressante. Tout d'abord, 
en quelques pages, Saussure récapitule l'essentiel de la critique exercée sur le moi par la philosophie hindoue. Selon son interprétation, c'est une critique du moi-veille par rapport au moi-sommeil en écartant l'Univers comme ne convenant pas à l'un de ces deux moi. Il présente d'abord le procédé philosophique de l'esprit hindou en deux points: 1 . écarter le monde et les sensations qui en proviennent, et 2 . assimiler ce moi sans qualités ni affections possibles à la substance universelle. Par la suite, il distingue deux variantes du deuxième point: l'idée bouddhique qui nie absolument le moi, et la philosophie sānkhya qui multiplie les moi (dans Parret, 1993: 221). Ce qui est ici significatif, c'est que Saussure dresse une typologie indienne du moi en trois catégories, répondant à la préoccupation des trois états physiologiques qui forment l'une des distinctions matérielles primordiales: veille, sommeil avec rêve (svapna), sommeil sans rêve (nidrā).

Ainsi, il repère les trois vérités fondamentales de cette philosophie: 1. l'être final est la substance universelle immuable; 2 . à cet instant, le moi et le non-moi qui se dégagent de tous les phénomènes se confondent; 3. la conscience du moi est classée comme phénomène. Dans cette condition du moi final ou de l'Être universel, il indique une conséquence singulière du corollaire physiologique selon lequel le moi parfait et le principe universel parfait se trouvent dans l'homme qui dort sans rêve. Voici son commentaire:

[...] l'état où se trouve le moi dans l'état psychique du sommeil sans rêve doit représenter la pureté du moi, puisque c'est dans ce seul état qu'il ne hui parvient rien de «l'extérieur».

(Ibid.: 223) ${ }^{3}$

Après son interprétation de la philosophie critique du moi faite par les Hindous, Saussure lui-même manifeste un avis favorable à cette critique dans une métaphore intéressante:

À travers buissons/N'est-il pas ridicule et même intolérable

d'être constamment enfermé dans son moi particulier et assujetti à ce tout petit moi? Je donnerais bien peu pour connaître "les sentiments d'Octave après la bataille d'Actium" [...] mais tout pour avoir été pendant trois minutes Octave luimême, soit avant soit longtemps avant cette bataille. (Ibid.)
En dernier ressort, Saussure envisage de caractériser le conflit fondamental entre l'Inde et la pensée occidentale comme suit:

Pour cette dernière la question s'est posée séculairement entre

le moi, comprenant ses sensations, et le non-moi / pour l'Inde,

éternellement, entre le non-moi et le moi en excluant du moi

les sensations elles-mêmes, comme non différentes de l'objet.

(Ibid.: 222)

\section{LÉVI-STRAUSS ET LE BOUDDHISME:} QUELQUES ASPECTS DE LA RÉCEPTION

Il est bien connu que Lévi-Strauss a montré à maintes reprises une attitude tout à fait positive à l'égard du bouddhisme. Mais, comme nous l'avons dit précédemment, les études approfondies sur la réception de la pensée bouddhique font défaut chez le fondateur de l'anthropologie structurale. J'ai consulté une dizaine d'ouvrages lui étant consacrés sans rien trouver de spécifique à ce sujet ${ }^{4}$. Il existe néanmoins trois études remarquables dans le milieu anglophone, qui ont tenté un rapprochement entre les deux univers de pensée à la fois si lointains et si proches. En affirmant la valeur théorique et historique d'un tel parallélisme, Strenski énonce qu' «un esprit commun semble à l'œuvre dans ces deux réalités historiques apparemment différentes" (1980: 3; notre traduction) $)^{5}$.

Au niveau théorique, il justifie un tel travail comparatif par leur idéologie universelle de la nature humaine. Selon lui, le bouddhisme et le structuralisme présentent quelques aspects de l'une des grandes options idéologiques que les sciences humaines doivent essayer de comprendre.

Octavio Paz a souligné, pour sa part, la nécessité historique de la similarité philosophique entre le bouddhisme et la pensée structuraliste de LéviStrauss, et l'universalité de la pensée humaine. Selon lui, ce n'est pas un hasard si l'on découvre des similarités entre le bouddhisme et la pensée de LéviStrauss:

[...] c'est une preuve supplémentaire que l'Occident, par ses moyens propres, et par la propre logique de son histoire, aboutit aux conclusions fondamentalement identiques à celles de Bouddha et ses disciples. La pensée humaine est une. (Paz, 1970: 151-152; notre traduction) ${ }^{6}$ 
Enfin, un autre interprète anglais a bien repéré des points communs ou des liens profonds entre les deux chemins de pensée dans le contexte de la critique morale et écologique de Lévi-Strauss, vis-à-vis de l'humanisme occidental, et dans son refus radical de l'idéal du progrès et du matérialisme (Pace, 1986: 198).

Avant d'essayer de faire un rapprochement entre le bouddhisme et la pensée structuraliste de Lévi-Strauss, il est nécessaire de procéder à un repérage historique. Dans cette optique, il semble indispensable de connaître la profondeur et la nature de l'orientalisme qui recouvre une partie importante de la pensée française du XXe siècle. Il est tentant de voir cette affinité de la pensée structuraliste avec le bouddhisme dans la situation politique et sociale de Lévi-Strauss qui, en tant que Juif sous la IIIe République, avait des penchants pour la gauche. Mais on se demande aussi quelles sont les sources du bouddhisme de Lévi-Strauss. Il y a lieu de rappeler ici une grande tradition française de recherches indiennes du début du XIXe au XXe siècle - de Burnouf à Filliozat - et de focaliser sur le grand indianiste français Sylvain Lévi, qui a formé et diffusé une attitude humaniste dans l'élite intellectuelle et spirituelle de la France à l'époque où le jeune anthropologue faisait ses études. Cette attitude humaniste a été adoptée par un certain nombre de grands orientalistes français (voir Lubac, 1952: 262-285).

La France, nourrie de la tradition classique, cherche l'esprit humain à travers l'espace et le temps. Elle s'intéresse à l'Inde comme elle s'intéresse à la Chine. [...] l'indianisme français est surtout attiré par le bouddhisme, la seule production du génie indien qui soit d'ordre universel par son inspiration et par son histoire. (Lévi, 1926: 125)

Mais un historien a critiqué avec justesse cette attitude humaniste en indiquant un défaut déterminant: [...] ce que M. Sylvain Lévi en apprécie, ce n'est pas ce qu'ils pouvaient contenir et répandre de vérité: c'est leur importance comme facteurs de civilisation. Il décrit et goûte «l'humanisme asiatique" qu'ils ont suscité. Il ne se demande pas ce que vaut, dans l'absolu, la réponse apportée par le bouddha Sakyamouni et par ses disciples aux questions que fait lever en l'homme le mystère des choses et l'angoisse de son destin [...].

(Lubac, 1952: 263)
Abordons à présent la réception du bouddhisme dans l'œuvre de Lévi-Strauss. C'est d'abord dans Tristes Tropiques que l'on trouve différents passages - au total cinq - permettant d'évaluer les attitudes extrêmement favorables, voire élogieuses, de LéviStrauss à l'égard du bouddhisme. Le bouddhisme se reflète dans les images de la féminité et du pacifisme en contraste avec celles de l'Islam et du christianisme: «[...] le bouddhisme conçoit plutôt cette quiétude comme une fusion: avec la femme, avec l'humanité, et dans une représentation asexuée de la divinité» (1955: 436). Il suggère une autre métaphore comparative et contrastée du Sage et du Prophète:

Ni l'un ni l'autre ne sont des dieux, voilà leur unique point commun. À tous autres égards ils s'opposent: l'un chaste, l'autre puissant avec ses quatre épouses; l'un androgyne, l'autre barbu; l'un pacifique, l'autre belliqueux; l'un exemplaire et l'autre messianique. (Ibid.: 436-437)

Enfin, Lévi-Strauss relève les cinq points essentiels de la philosophie bouddhiste, qui se résument, d'après nous, en négation radicale ou immanentisme radical: l'unification du connaitre et de l'être, la dissolution de la distinction du sens et du non-sens ou le refus absolu du sens, selon son expression, le transfert du problème métaphysique à celui de la conduite humaine et ${ }^{7}$, enfin, le refus absolu du moi. Voici les affirmations lévi-straussiennes à ce propos:

Il n'y a pas d'au-delà pour le bouddhisme; tout s'y réduit à une critique radicale, comme l'humanité ne devait plus jamais s'en montrer capable, au terme de laquelle le sage débouche dans un refus $d u$ sens des choses et des êtres: discipline abolissant l'univers et qui s'abolit elle-même comme religion. (Ibid.: 437)

Tout effort pour comprendre détruit l'objet auquel nous nous étions attachés, au profit d'un effort qui l'abolit au profit d'un troisième et ainsi de suite jusqu'à ce que nous accédions à l'unique présence durable, qui est celle où s'évanouit la distinction entre le sens et l'absence du sens: la même d'où nous étions partis. [...]

Cette grande religion $d u$ non-savoir ne se fonde pas sur notre infirmité à comprendre. Elle atteste notre aptitude, nous élève jusqu'au point où nous découvrons la vérité sous forme d'une exclusion mutuelle de l'être et du connaître. Par une audace supplémentaire elle a - seule avec le marxisme - ramené le 
problème métaphysique à celui de la conduite humaine. [...]

Le moi n'est pas seulement haïssable: il n'a pas de place entre un nous et un rien. (Ibid.: 441)

Il énonce alors une convergence surprenante entre le marxisme et le bouddhisme dans un passage très célèbre:

Entre la critique marxiste qui affranchit l'homme de ses premières chaînes - lui enseignant que le sens apparent de sa condition s'évanouit dès qu'il accepte d'élargir l'objet qu'il considère - et la critique bouddhiste qui achève la libération, il n'y a ni opposition ni contradiction. (Ibid.: 442) ${ }^{8}$

Dans le dialogue entre la pensée structuraliste de Lévi-Strauss et la pensée bouddhique, il faut s'engager dans un travail de transformation mutuelle, basé sur un comparatisme constructif, ne se limitant pas à dresser un tableau comparatif, autour des notions bouddhistes telles que l'interdépendance, la non-substantialité ou la causalité. Néanmoins, on peut tenter un rapprochement conceptuel. Je voudrais évoquer les trois points communs entre le structuralisme de Lévi-Strauss et le bouddhisme que sont la pensée relativiste ou décentraliste, la pensée écologique et la déconstruction du sujet. Après une présentation rapide des deux premiers, nous examinerons le troisième plus en détail.

En premier lieu, notre anthropologue structuraliste a travaillé sans cesse à décentraliser l'homme. Comme le bouddhisme nous l'a appris, pour lui, l'esprit de l'Homme n'est qu'un produit de la Nature, ne lui est pas extérieur. Il y a une continuité entre l'homme observant et l'objet analysé. Il affirme dans le dernier passage des Tristes Tropiques cette décentralisation de l'homme dans l'univers: «Le monde a commencé sans l'homme et s'achèvera sans lui». En deuxième lieu, Lévi-Strauss et le Bouddha partagent la vision écologique. Selon eux, l'homme est un organisme vivant parmi d'autres. Dans cette condition, il faut bien admettre que ses droits ne se justifient que par une cohabitation juste avec d'autres espèces vivantes dans un respect strict dû à l'espèce en tant qu'espèce et donc dû à toutes les espèces (1983: 376):

En isolant l'homme du reste de la création, en définissant trop étroitement les limites qui l'en séparent, l'humanisme occidental hérité de l'Antiquité et de la Renaissance l'a privé d'un glacis protecteur [...] on avait oublié que, si l'homme est respectable, c'est d'abord comme être vivant plutôt que comme seigneur et maître de la création: première reconnaissance qui l'eût contraint à faire preuve de respect envers tous les êtres vivants. À cet égard, l'Extrême-Orient bouddhiste reste dépositaire de préceptes dont on souhaiterait que l'humanité dans son ensemble continuât ou apprît à s'inspirer. (Ibid.: 46)

Dans son ouvrage monumental, La Pensée sauvage, l'anthropologue demande de réintégrer la culture dans la nature, et ultérieurement la vie humaine dans l'ensemble de ses conditions naturelles. Ainsi, il souligne la continuité entre les propriétés de l'esprit et celles du monde et renie un certain réalisme cognitif indépendant de l'environnement. Lévi-Strauss anticipe en quelque sorte la notion de cognition incarnée élaborée par Varela qui, à maintes reprises, a évoqué un rapprochement entre ses théories cognitives et le bouddhisme. Il déclare dans l'Inscription corporelle de l'esprit que «la cognition, loin d'être la représentation d'un monde prédonné, est l'avènement conjoint d'un monde et d'un esprit à partir de l'histoire des diverses actions" (1993: 35). Autrement dit, «la connaissance ne préexiste pas en un seul lieu ou en une forme singulière, elle est chaque fois énactée dans des situations particulières» (ibid.: 37).

Lévi-Strauss nous a souvent rappelé l'indifférence bouddhique, la grande tolérance des moines bouddhistes et le détachement. Il est fort probable qu'il se soit inspiré des idées écologiques et pacifiques du bouddhisme, telles que le rien, la contemplation, l'impassibilité. Aux yeux du Bouddha, chaque espèce vivante a sa propre valeur dans la nature et l'homme n'a aucun privilège par rapport aux autres espèces. Le dernier passage de Tristes Tropiques consacré à l'observation et à la description d'un paysage rocheux est pour moi un beau poème écologique.

Enfin, il nous reste à aborder la question de la dissolution du sujet. L'anthropologue structuraliste a repéré dans le culte humaniste du sujet un vice rédhibitoire de la culture occidentale. Pour lui, le sujet est comme «un insupportable enfant gâté qui a occupé trop longtemps la scène philosophique 
et empêche tout travail sérieux en réclamant une attention excessive» (1971: 615). Il a nié un humanisme usurpateur qui établit l'Homme comme un seigneur absolu de la création, puisque la dignité exclusive de l'homme a provoqué à la nature une première amputation.

Il avait déjà affirmé dans La Pensée sauvage: «Le but dernier n'est pas de constituer l'homme mais de le dissoudre» (1962: 295). Cette dissolution n'est pas autre chose qu'impermanence ou fugacité, souffrance et irréalité du Moi, qui sont les trois caractéristiques de l'être humain selon le bouddhisme.

Au regard des règles et des faits culturels très complexes comme les rapports de parenté et les mythes, Lévi-Strauss affirme, suivant l'enseignement de la linguistique structurale, que les structures sémiotiques ne proviennent ni d'un sujet transcendantal anhistorique, ni des sujets agissants qui les pratiquent, mais d'une vie autonome du symbolique.

Cependant, dans le "finale" de L'Homme nu de Lévi-Strauss, qui annonce à la fois la dissolution du sujet et la constitution positive de la subjectivité, il faut relever deux paradoxes (Keck, 2004). Le premier est qu'il semble introduire la transcendance d'un sujet dans l'immanence du tissu mythique. Un mythe dispose du trait distinctif de venir de plus loin que le sujet. Il nous fait comprendre que l'effacement du sujet représente une nécessité d'ordre méthodologique pour garder une explication autonome du mythe par le mythe. Le second paradoxe peut s'exprimer par la question suivante: comment un sujet peut-il émerger au terme de ce procès de dissolution dans les mythes? La réponse est qu'il s'agit de constater l'arrêt momentané de cet effacement du sujet et la présence irréductible d'un résidu qui correspond au moi selon lui.

Cette idée s'observe dans la conception bouddhique de l'absence du moi (anātman) qui distingue le plus nettement le bouddhisme des autres doctrines philosophiques par la radicalité de cette position. Voici un commentaire éclairant:

[...] ce qui dit ou pense "je» n'est pas un sujet - n'en déplaise à Descartes. Le moi tel que nous le concevons d'ordinaire est une illusion, un produit de l'ignorance fondamentale et de toute une série de causes et d'effets - la causalité karmique. [...] On ne trouve, derrière tous les états émotionnels ou psychiques, les pensées et les actions, aucun principe fédérateur ou unificateur immuable qu'on pourrait qualifier de moi.

(Berval, 1987: 170-171)

Le sujet comme foyer virtuel où viennent se croiser les rayons constitutifs du réel devient momentanément un moi réel.

Le sujet se dissout dans les mythes comme l'humanité se dissoudra un jour dans une grande explosion astrale; avant ce moment final, il n'y a que des croisements singuliers d'étoiles et des résidus de sujets. (Lévi-Strauss, 1971: 494)

En résumé, il faut saisir le double aspect du sujet chez lui. Il y a dans ces réflexions critiques, de la part de Lévi-Strauss, une invitation à déconstruire la notion même de sujet. Le sujet consacre ainsi un lieu insubstantiel et vide auquel le structuralisme a su renoncer, «mais aussi un lieu plein et vivant de sens auquel le super-rationalisme de Lévi-Strauss donnerait en bout de course un accès» (Cazier, 2007: 176).

L'idée lévi-straussienne de la dissolution du sujet s'accorde avec la notion du sujet distribué de Varela qui affirme que le sujet ayant la conscience ne siège pas dans un centre, qu'il soit neuronal ou métaphysique, mais réside à même notre inscription dans les environnements de notre existence. Il s'agit donc d'un esprit distribué dans le monde, non plus encapsulé dans une forme d'intériorité de la conscience $^{9}$. Dans une telle conception distribuée et fluide de l'esprit et du moi, le moi se trouve dans l'autre. L'existence s'accomplit sur le mode de la distribution, de la médiation et du décentrement. Notons au passage que, pour Lacan, ce moi massif et tout-puissant n'existe pas non plus: pour lui, il est impossible que le sujet soit identique à quelque chose qui serait lui-même, puisque le sujet est défini comme sujet du fantasme. Notre identité nous échappe, car nous sommes le fantasme de l'autre.

Dans la théorie de Varela, le moi serait non pas un élément stable, continu, ontologiquement délimité, mais une simple propriété émergente de structures sous-jacentes indépendantes, dotées d'une existence non pas réelle, mais purement nominale. 
Oublier son moi et devenir complètement quelque chose, c'est aussi prendre conscience de sa propre vacuité, c'est-à-dire de l'absence de point de référence solide. (Varela, 1996: 59)

\section{CONCLUSION}

Dans l'introduction du présent article, nous parlions de la difficulté de mener une étude complète sur les liens épistémologico-historiques du bouddhisme et du structuralisme. En effet, nous avons constaté une quasi-absence d'études approfondies traitant le retentissement et l'ampleur que le bouddhisme a eus dans les sciences humaines françaises du siècle dernier. Nous avons néanmoins effectué un comparatisme constructif en vue de révéler quelques traces d'influence et d'affinité entre ces deux chemins de pensée universelle. Il s'agissait tantôt d'analogies frappantes, tantôt de rapprochements à l'échelle profonde. L'accent mis sur les convergences entre ces deux réalités historiques découle de la volonté de pratiquer le comparatisme assimilateur qui valorise des similitudes au lieu du comparatisme contrastif focalisant sur les dissemblances. Je n'ai pas voulu donner dans cette contribution une présentation systématique du bouddhisme et de la pensée structuraliste; mon propos a plutôt été de me concentrer sur certains thèmes saillants posés autour des fondations métaphysiques de deux grands penseurs modernes à la lumière de quelques élémentsclés de la réflexion bouddhique que sont la vacuité, la critique du moi et la philosophie écologique ou le décentrement de l'anthropocentrisme tout court.

\section{NOTES}

1. Si l'on compare ce commentaire avec celui d'un bouddhologue contemporain, on s'aperçoit que la compréhension saussurienne de la nature de la pensée bouddhisme est pénétrante. «L'alternative scolaire - philosophie ou religion? - n'a guère de sens en ce qui concerne un bouddhisme par nature pluriel. Philosophie et religion ne peuvent être maintenues séparées que selon une perspective ethnocentrique, dont précisément la pensée non dualiste du bouddhisme nous permet de nous détacher " (Faure, 1998: 113).

2. On trouve des passages similaires qui manifestent le doute saussurien sur l'existence de l'identité: «Où est maintenant l'identité? On répond en général par un sourire, comme si c'était une chose en effet curieuse (sans) remarquer la portée philosophique de la chose [...]. Où est réellement l'identité? Je pourrais fort bien dire que ce qui est c'est chose bien plus incalculable qu'il aurait été si nous avions essayé de vouloir la fonder sur quelque chose, mais du même coup sur quoi que ce soit, même sur une combinaison de caractères" (ibid.).

3. Selon Borges, il s'agit de l'énigme du nirvana: «L'énigme du nirvana est peut-être identique à l'énigme du sommeil; dans les Upanishads on lit que les hommes plongés dans un sommeil profond sont l'univers.

Selon Sankyyam, l'état de l'âme en profond sommeil est le même que celui qu'elle atteindra après sa délivrance" (1979: 74).

4. J.-P. Cazier, 2007; M. Izard, 2008; M. Hénaff, 2008; E. Désveaux, 2008.

5. "a common 'mind' may be seen at work in theses two apparently different historical realities."

6. "I will say finally that the similarity between Buddhism and LéviStrauss's thought is not accidental: it is one more proof that the West, by its own means, and by the very logic of its history, is now arriving at conclusions fundamentally identical to those Buddha and his disciples had arrived at. Human thought is one, and we owe it to Lévi-Strauss

- among many other things - to have demonstrated that the reason of the primitive or of the Oriental is no less rigorous than our own."

7. A ce sujet, Conze partagerait cet aspect avec Lévi-Strauss: «le bouddhisme, en tant que philosophie, peut être défini comme un "pragmatisme dialectique" avec une tendance "psychologique" (1975: 15; notre traduction). "Buddhism, as a 'philosophy' could then be described as a 'dialectical pragmatism' with a 'psychological' turn." 8. Dans ce passage, Lévi-Stauss signale cette jonction en Rousseau du bouddhisme schopenhauerien (stratégie de la pitié) et de l'humanisme marxiste (critique de la représentation aliénée). Voir O. Abel, Postface au structuralisme anthropologique de Lévi-Strauss, 1984. En ligne: http:// olivierabel.fr/ (page consultée le 21 avril 2011).

9. À ce sujet, je me réfère à l'article de M.-A. Paveau, «Un sujet distribué. Lacan, Bouddha, Varela", La Pensée du discours, 2010. En ligne: http://penseedudiscours.hypotheses.org/2527 (page consultée le 21 avril 2011). 


\section{RÉFÉRENCES BIBLIOGRAPHIQUES}

AMACKER, R. [1988] : «Notules II. Compléments à la "Bibliothèque de Saussure" ", Cahiers Ferdinand de Saussure, no 42, 245-248.

ARRIVÉ, M. [2007]: À la recherche de Ferdinand de Saussure, Paris, PUF. Atlani-Voisin, F. [2003]: «Le don de l'Inde», dans S. Bouquet (dir.), 79-93.

BerVAL, R. de (dir.) [1987]: Présence du bouddhisme, Paris, Gallimard. BONARDEL, F. (dir.) [2008]: «Existence et vacuité selon Sartre et le bouddhisme zen ", Bouddhisme et philosophie, Paris, L'Harmattan, 193. 218.

Borges, J. L. et A. JuRADO [1979]: Qu'est-ce que le bouddhisme?, Paris, Gallimard.

BOUQUET, S. (dir.) [2003]: Saussure, Paris, Éd. de L'Herne, coll.

"Cahiers de L'Herne" n॰ 76.

BURNOUF, E. [(1844) 1876] : L'Introduction à l'histoire du bouddhisme indien, Paris, Imprimerie Royale.

CAZIER, J.P. (dir.) [2007]: Abécédaire de Claude Lévi-Strauss, Mons, Sils Maria, coll. " Abécédaire n० 4 ".

CHENET, F. [2005]: «Du sens de la philosophie comparée», dans J. Lacrosse et alii (collectif), Philosophie comparée: Grèce, Inde, Chine, Paris, Vrin, 79-97.

CONZE, E. [(1951) 1975]: Buddhism: its Essence and Development, New York, Harper Torchbooks.

D'OtTAVI, G. [2010] : «Saussure et l'Inde: la théorie de l'apoha et les entités négatives du langage », dans C. Bota, J.-P. Bronckart et E. Bulea (dir.), Le Projet de Ferdinand de Saussure, Genève, Droz, 161-191.

DÉSVEAUX, E. [2008]: Au-delà du structuralisme, Paris, Éd. Complexe. DroIT, R.P. [1989]: L'Oubli de l'Inde: une amnésie philosophique, Paris, PUF;

- [1997]: Le Culte du néant: les philosophes et le bouddha, Paris, Seuil. ENGLER, R. (dir.) [(1968) 1989]: Ferdinand de Saussure. Cours de linguistique générale, édition critique tome 1, Wiesbaden, Harrassowitz; (dir.) [(1974) 1990]: Ferdinand de Saussure. Cours de linguistique générale, édition critique tome 2: Appendice, Notes de F. de Saussure sur la linguistique générale, Wiesbaden, Harrassowitz;

[1974-1975]: «Sémiologies saussuriennes», Cahiers Ferdinand de Saussure, $n^{\circ} 29$, 45-73.

FAURE, B. [1998]: Bouddhisme, philosophies et religions, Paris, Flammarion.

FEHR, J. [1997]: Saussure entre linguistique et sémiologie, Paris, PUF. GODEL, R. [1957a]: «Cours de linguistique générale (1908-1909). Introduction (d'après des notes d'étudiants)", Cahiers Ferdinand de Saussure, no 15, 3-103;

[1957b]: Les Sources manuscrites $d u$ Cours de linguistique générale, Genève et Paris, Droz et Minard.

HÉnAFF, M. [2008]: Claude Lévi-Strauss, le passeur de sens, Paris, Perrin. IZARD, M. (dir.) [2004]: Lévi-Strauss, Paris, Éd. de L'Herne, coll. "Cahiers de L'Herne" n 82.

Jullien, F. [1995]: Le Détour et l'Accès: stratégies du sens en Chine, en Grèce, Paris, Grasset;

[2005] : « La Grèce et la Chine: comparer, dé-comparer. Un entretien avec François Jullien ", dans J. Lacrosse et alii (collectif), 65-78.
KECK, F. [(2004) 2008]: « La dissolution du sujet dans le "finale" de L'Homme nu», dans M. Izard (dir.), 214-220.

LACROSSE, J. et alii (collectif) [2005]: Philosophie comparée: Grèce, Inde, Chine, Paris, Vrin.

LAMOTTE, E. [1976]: Histoire du bouddhisme indien: des origines à l'ère Sàka, Louvain-la-Neuve, Presses de l'Université de Louvain.

LÉVI, S. [1926]: L'Inde et le Monde, Paris, Honoré Champion. LÉVI-STRAUSS, C. [1955]: Tristes Tropiques, Paris, Plon;

[1962]: La Pensée sauvage, Paris, Plon;

_ [1964]: Mythologiques I. Le Cru et le Cuit, Paris, Plon;

_ [1971]: Mythologiques IV. L'Homme nu, Paris, Plon;

[1983]: Le Regard éloigné, Paris, Plon.

LuBAC, H. de [1952]: La Rencontre du bouddhisme et de l'Occident, Paris, Aubier.

MARINETti, A. et M. MeLi (dir.) [1986]: Ferdinand de Saussure. Le leggende germaniche, édition des manuscrits mythographiques, Este, Zielo.

Nietzsche, F. [1993]: CEuvres complètes, 2 tomes, Paris, Robert Laffont. OBADIA, L. [1999]: Bouddhisme et Occident, Paris, L'Harmattan.

Oltremare, P. [1906]: L'Histoire des idées théosophiques dans l'Inde, Paris, Annales du Musée Guimet.

PACE, D. [1986]: Claude Lévi-Strauss: The Bearer of Ashes, Londres, ATK Paperbacks.

PARRET, H. [1993] : «Les manuscrits saussuriens de Harvard », Cahiers Ferdinand de Saussure, no 47, 179-234;

_- [2003]: «Métaphysique saussurienne de la voix et de l'oreille dans les manuscrits de Genève et de Harvard ", dans S. Bouquet (dir.) 64-80.

PAZ, O. [1970]: Claude Lévi-Strauss: An Introduction, Ithaca et Londres, Cornell University Press.

RASTIER, F. [2002]: «Saussure, la pensée indienne et la critique de l'ontologie ", Revue de sémantique et de pragmatique, $\mathrm{n}^{\circ}$ 11, 123-146;

_ [2003]: «Le silence de Saussure ou l'ontologie refusée», dans S. Bouquet (dir.), 25-53.

SAUSSURE, F. de [(1972) 1995]: Cours de linguistique générale, introduction, traduction et commentaire de T. De Mauro, Paris, Payot, I-XVIII et 319-495;

[2002]: Écrits de linguistique générale, textes établis par S. Bouquet et R. Engler, avec la coll. d'A. Weil, Paris, Gallimard.

SMALL, E. [1987]: «Semiotic referentiality: Saussure's sign and the sanskrit nama-rupa ", The American Journal of Semiotics, vol. 5, no 3/4, 447-459.

STRENSKI, I. [1980]: «Lévi-Strauss and the buddhists», Comparative

Studies in Society and History, vol. 22, $\mathrm{n}^{\circ} 1,3-22$.

SuZUKI, D. T., E. Fromm ET R. DE MARTINO [1971]: Bouddhisme et Psychanalyse, Paris, PUF.

VARELA, F. [(1992) 1996]: Quel savoir pour l'éthique? Action, sagesse et cognition, Paris, La Découverte.

VARELA, F., E. THOMPSON et E. ROSCH [(1991) 1993]: L'Inscription corporelle de l'esprit, Paris, Seuil.

WANG, Y. [2001]: Buddhism and Deconstruction: Towards a Comparative Semiotics, Richmond, Curzon Press. 